\title{
Automated Crystal Orientation Mapping with a Liquid-Cell TEM
}

Eric Lang ${ }^{1}$, Caitlin Taylor ${ }^{2}$, Sarah Pratt ${ }^{3}$, Tina Nenoff ${ }^{3}$ and Khalid Hattar ${ }^{3}$

${ }^{1}$ Sandia National Laboratories, Albuquerque, New Mexico, United States, ${ }^{2}$ Los Alamos National Laboratory, United States, ${ }^{3}$ Sandia National Laboratories, United States

Rare earth metals and their compounds are used extensively in renewable energy and computing systems, and found in nuclear power plant waste streams. However, their low supply and high demand means they must be produced efficiently, safely, and ultimately recycled for reuse [1]. Processing at room temperature presents a fabrication pathway that meets these demands, but the evolution of the structure and chemistry during these processes is not well established. One room temperature processing route for lanthanide nanoparticles is gamma irradiation radiolysis synthesis [2]. In this process, hydrogen atoms, hydroxyl radicals, and electrons are generated from $\mathrm{H} 2 \mathrm{O}$ in the solution. The liberated electrons can then reduce the metal ions to produce stable metal nanoparticles.

To study the radiolysis process, a system must be able to analyze the structure and composition of metal nanoparticles in an aqueous solution under irradiation in real time. Liquid cell TEM stages offer this opportunity [2-6]. In radiolysis in microfluidic TEM stages, water containing nanoparticles of interest flows through a stage while the electron beam introduces the free electrons that can reduce the metal ions $[2,3]$. Microfluidic stages have been used previously to study nanostructure nucleation and growth in other systems [5-10].

The morphology, phase, and orientation of materials dictate material properties and function in synthesis and fabrication of rare earth metal compounds. To map the orientation in the TEM, Precession Electron Diffraction (PED) can be used to produce Automated Crystal Orientation Maps (ACOM) utilizing the ASTAR collection and analysis system (NanoMEGAS, Inc.). This process deconvolutes the diffraction patterns from regions of the sample to map the grain structure with high spatial resolution. Liquid cells represent a unique challenge for PED data collection. The beam-sensitivity, stability of samples in solution and, the thickness of the amorphous microfluidic cell limit the obtainable resolution collected.

In this study, we use electron beam irradiation-induced radiolysis to nucleate metal nanoparticles. In-situ imaging with the microfluidic stage, while monitoring the electron beam flux and size allowed for analysis of the nanostructure nucleation avenues and rates. In this work, rare earth salt aqueous solutions of yttrium (III) nitrate hydrate, lanthanum (III) chloride hydrate, and europium (III) chloride hydrate were prepared and analyzed in a JEOL 2100 TEM. Using a microfluidic TEM stage (Protochips, Inc.), the solutions were trapped between two $50 \mathrm{~nm}$-thick SiNx windows, and used in both open cell and closed cell modes (with/without external flow between an outside reservoir and the cell). Crystallization of nanoparticles was tracked in real-time as the electron beam was converged and diverged to introduce high electron doses to the solution to induce the radiolysis and nucleation of nanoparticles [11]. Following nucleation, ACOM was performed to map the nanoparticle orientations. The results show that the TEM electron beam-induced radiolysis results in reduction, oxidation, and hydride formation of the aqueous rare earth salt solutions for lanthanum (III) chloride hydrate and europium (III) chloride hydrate. This work also demonstrates the use of ACOM for future mapping in liquid cells to understand nanocrystalline orientation relationships in liquid environments which presents many future research prospects. While challenges were encountered in this first attempt, we show that ACOM can provide useful insights into the orientation and crystallinity of nanostructures grown in aqueous solutions in the TEM. 


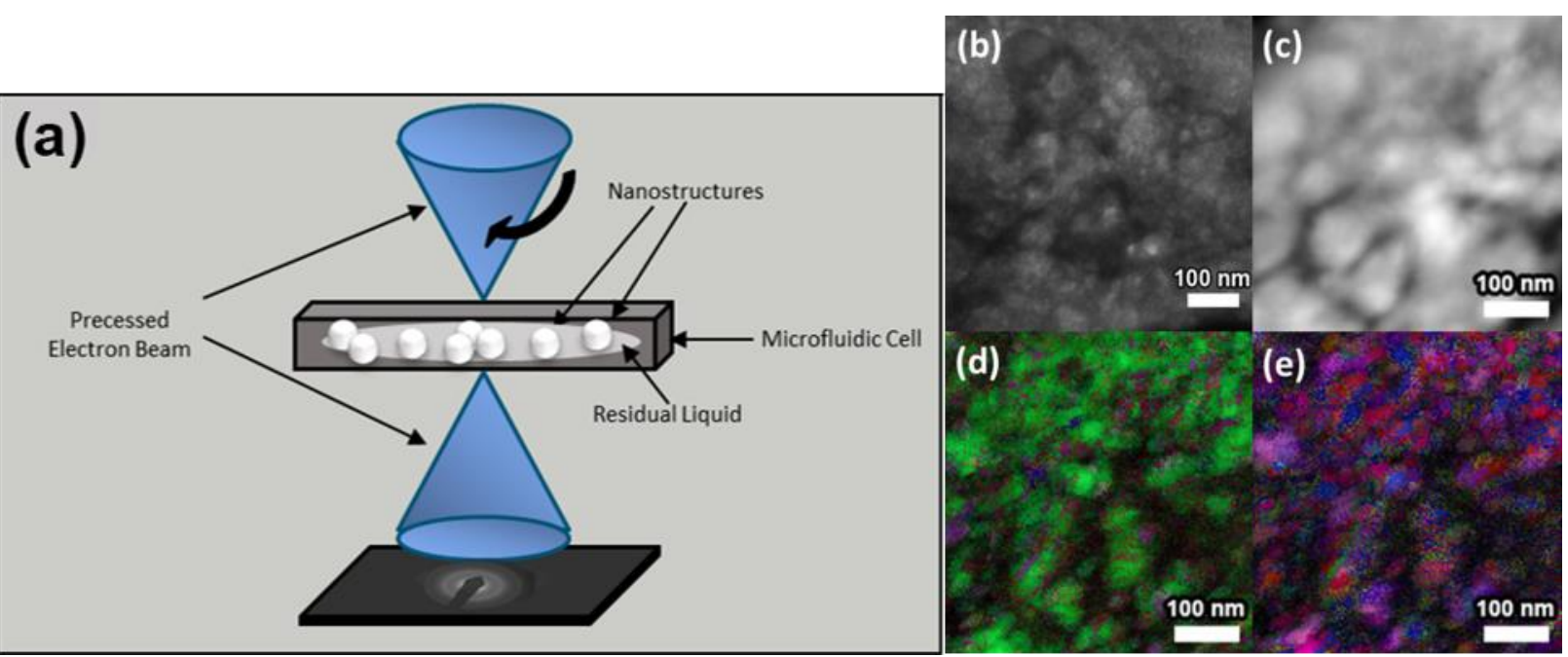

Figure 1. Fig. 1: (a) Schematic of the PED process of a precessing electron beam through the microfluidic cell with liquid and nanoparticles with the collection of diffraction patterns on the bottom screen with a $\mathrm{LaCl}$ - 7H2O diffraction pattern. (b-e) PED scans of La metal nanostructures formed during electron beam irradiation. (b) BF-TEM micrograph of the region of interest, (c) virtual BF image produced by ACOM software, ACOM maps of Y orientation (d) and Z orientation (e) of La metal crystals collected from the area in (c).

\section{References}

[1] D. Bauer, et al. Critical Materials Strategy, U.S. Department of Energy, 2010.

[2] J. Grand, et al. The Journal of Physical Chemistry C, 122 (2018), 12573-12588.

[3] L. R. Parent, et al. ACS Nano, 6 (2012), 3589-3596.

[4] T. Prozorov, et al. Journal of the Royal Society Interface, 14 (2017), 20170464.

[5] J. E. Evans, et al. Nano Letters, 11 (2011), 2809-2813.

[6] N. Ahmad, et al. ACS Nano, 13 (2019), 13333-13342.

[7] O. Roth, et al. Journal of Nuclear Materials, 383 (2009), 231-236.

[8] A. Kobler, et al. Ultramicroscopy, 173 (2017), 84-94.

[9] T.J. Woehl, et al. ACS Nano, 6 (2012), 8599-8610.

[10] M.H. Nielsen, et al. Microscopy \& Microanalysis, 20 (2014), 425-436.

[11] C.A. Taylor, et al. Nanoscale Advances, 1 (2019), 2229.

This work was supported by the US Department of Energy, Office of Basic Energy Sciences. Sandia National Laboratories is a multi-mission laboratory managed and operated by National Technology and Engineering Solutions of Sandia, LLC., a wholly owned subsidiary of Honeywell International, Inc., for the U.S. Department of Energy's National Nuclear Security Administration under contract DE-NA0003525 . 